\title{
Short communication: A sensitive method for qualitative screening of bile salt hydrolase-active lactobacilli based on thin-layer chromatography
}

\author{
C. F. Guo, ${ }^{,}$L. W. Zhang, ${ }^{* 1}$ X. Han, ${ }^{\star}$ J. Y. Li, ${ }^{,}$M. Du, ${ }^{*}$ H. X. Yi, ${ }^{*}$ Z. Feng,, Y. C. Zhang, ${ }^{\star}$ and X. R. Xu \\ *School of Food Science and Engineering, Harbin Institute of Technology, Harbin, Heilongjiang 150090, China \\ †College of Food Science, Northeast Agricultural University, Harbin, Heilongjiang 150030, China
}

\begin{abstract}
A sensitive protocol based on thin-layer chromatography (TLC) was developed to screen qualitatively bile salt hydrolase (BSH)-active lactobacilli. The sodium salts of glycocholic acid and taurocholic acid were used as substrates, and bacterial BSH activity was confirmed by detecting cholic acid as a product of the bile conjugates using a TLC assay with direct visual observation. Forty-five lactobacilli isolated from human fecal samples were tested for BSH activity by the TLC assay, a conventional plate assay, and a quantitative colorimetric assay. With the TLC and quantitative colorimetric assays, the same $24 \mathrm{BSH}$-positive strains were detected. No false-positive or false-negative results were detected by the TLC assay. However, only 20 BSH-positive strains were detected with the conventional plate assay. Compared with the conventional plate assay, the TLC assay is more sensitive for the detection of BSH activity of lactobacilli and, thus, more suitable for screening of $\mathrm{BSH}$-active lactobacilli of human origin.
\end{abstract}

Key words: bile salt hydrolase, thin-layer chromatography, plate assay, lactobacilli

\section{Short Communication}

Bile salt hydrolase (BSH, EC 3.5.1.24), which is also called cholylglycine hydrolase, is an enzyme that catalyzes the hydrolysis of glycine- and taurine-conjugated bile acids into AA residues and free bile acids (Kim et al., 2004). Bile salt hydrolase acts upon a wide range of bile acid conjugates, including 6 major human conjugated bile acids (taurocholic acid (TCA), taurodeoxycholic acid (TDCA), taurochenodeoxycholic acid (TCDCA), glycocholic acid (GCA), glycodeoxycholic acid (GDCA), and glycochenodeoxycholic acid (GCDCA)]. To date, BSH activity has been detected only in bacteria, and is expressed primarily by several bacterial species of the gastrointestinal tract, such as

Received September 8, 2010.

Accepted December 19, 2010

${ }^{1}$ Corresponding author: lanweizhang@yahoo.com.cn
Bacteroides spp., Clostridium spp., Enterococcus spp., Bifidobacterium spp., and Lactobacillus spp. (Begley et al., 2006).

Lactobacilli are normal inhabitants of the human intestine, and have been often used as probiotic agents due to the wide range of consumer benefits associated with their consumption, such as the treatment and prevention of enteric infections, prevention of colorectal cancer, and treatment of irritable bowel syndrome (Lebeer et al., 2008). Certain strains of lactobacilli that possess BSH activity also have the capacity to decrease serum cholesterol levels in animals (De Smet et al., 1998; Nguyen et al., 2007). Thus, the ability to hydrolyze bile salts has often been included among the selection criteria for probiotic strains with cholesterollowering properties (Pereira et al., 2003).

Bile salt hydrolase activity has been quantified by measuring bile acid conjugates using HPLC (Corzo and Gilliland, 1999b) or free acids using spectrophotometry (Walker and Gilliland, 1993). The activity also can be measured quantitatively by measuring the amount of taurine and glycine that has been released from the bile acid conjugates using colorimetric assay (Liong and Shah, 2005a; Noriega et al., 2006). Although quantitative assays are reliable, these remain complicated and time-consuming. Therefore, a plate assay as a qualitative detection method has been widely used to mitigate labor intensity. The plate assay was developed by Dashkevicz and Feighner (1989) and TDCA was used as a substrate with this method. However, Certain Lactobacillus strains with BSH activity lack the ability to deconjugate TDCA (Christiaens et al., 1992; Moser and Savage, 2001), with the result that false-negative outcomes would occur when using the plate assay for detection of BSH activity in such strains.

Cole and Fuller (1984) reported that certain strains of lactobacilli were able to hydrolyze glycine-conjugated, but not taurine-conjugated bile acids. In contrast, Fang et al. (2009) found that certain strains of lactobacilli were able to deconjugate taurine-conjugated, but not glycine-conjugated bile acids. However, to our knowledge, no BSH-active strain of lactobacilli exists that cannot deconjugate either GCA or TCA. On the basis 
of this finding, we hypothesized that use of a mixture of GCA and TCA as substrates might provide a more sensitive method to screen BSH-active lactobacilli. The objectives of this study were to develop a sensitive method for qualitative screening of BSH-active lactobacilli using a mixture of GCA and TCA as substrates based on thin-layer chromatography (TLC) assay.

The 45 strains of lactobacilli used in this study were isolated from 27 human fecal samples using LAMVAB agar (Hartemink et al., 1997) and were identified on the basis of Gram staining, catalase reaction, and carbohydrate fermentation patterns using Biolog System (Biolog, Hayward, CA). Lactobacillus acidophilus ATCC 43121 was used as a positive control (Brashears et al., 1998) and Escherichia coli ATCC 25922 as a negative control (Begley et al., 2006). The strains were cultured in de Man, Rogosa, and Sharpe (MRS) broth and stored at $4^{\circ} \mathrm{C}$. Prior to testing, all strains were subcultured twice at $37^{\circ} \mathrm{C}$ for $18 \mathrm{~h}$.

For the TLC assay of BSH activity, overnight cultures were harvested by centrifugation at $8,000 \times g$ for $10 \mathrm{~min}$ at $4^{\circ} \mathrm{C}$, and the cell pellets were resuspended in a volume of Ringer's solution equal to that of the original broth. Subsequently, $1 \mathrm{~mL}$ of the bacterial cell suspension or distilled water (control) was added to 1 $\mathrm{mL}$ of a reaction mixture. The reaction mixture was prepared by dissolving MRS dehydrated media (54 $\mathrm{g} / \mathrm{L})$, sodium salt of GCA $(5.6 \mathrm{mM})$, and sodium salt of TCA $(2.4 \mathrm{~m} M)$ in sodium phosphate buffer $(0.2 \mathrm{M}, \mathrm{pH}$ 7.4) and adjusting the final $\mathrm{pH}$ to 7.4 with $5 \mathrm{M} \mathrm{NaOH}$. The mixtures were incubated anaerobically for $8 \mathrm{~h}$ at $37^{\circ} \mathrm{C}$. After incubation, the mixtures were evaporated by lyophilization and the residues were redissolved in 1 $\mathrm{mL}$ of methanol. Following centrifugation $(8,000 \times g$, $10 \mathrm{~min})$, the supernatant $(3 \mu \mathrm{L})$ was spotted onto TLC plates $(20 \mathrm{~cm} \times 20 \mathrm{~cm}$ Silica gel 60, Merck, Darmstadt, Germany). Standard solutions [sodium salt of GCA, TCA, and cholic acid (CA) in methanol, $5 \mathrm{~m} M$ each] were analyzed using an identical procedure. The TLC plates previously saturated with mobile phase vapor were developed at $18 \pm 1^{\circ} \mathrm{C}$. The mobile phase consisted of isoamyl acetate-propionic acid-n-propanolwater (40:30:20:10). All solvents used were of analytical grade, purchased from Merck. When the solvent front was 1 to $2 \mathrm{~cm}$ from the top edges of the plates $(3-4 \mathrm{~h})$, the plates were removed from the jars and dried in an oven at $110^{\circ} \mathrm{C}$ for $3 \mathrm{~min}$. Bile acids were located on the TLC plates by spraying with $10 \%$ (wt/vol) phosphomolybdic acid (Sigma-Aldrich, St. Louis, MO) in ethanol and heating at $110^{\circ} \mathrm{C}$ for $5 \mathrm{~min}$ (Hofmann, 1962). The BSH activity of a strain was regarded as positive when significantly robust CA spots appeared on the TLC plates as compared with that observed in the control.
To assess the sensitivity of the TLC assay, BSH activity was also tested with the conventional plate assay (Dashkevicz and Feighner, 1989) and the quantitative colorimetric assay (Tanaka et al., 2000). For the plate assay, overnight cultures were streaked on MRS agar plates supplemented with $0.5 \%$ (wt/vol) sodium salt of TDCA (Sigma-Aldrich). The plates were then incubated anaerobically for $72 \mathrm{~h}$ at $37^{\circ} \mathrm{C}$. The BSH activity of strains was regarded as positive when deoxycholic acid (DCA) precipitated in the agar medium below and around a colony. For the colorimetric assay, a human bile salt mixture $(9.4 \mathrm{mM})$ was used as a substrate. The mixture consisted of sodium salts of TCA, TCDCA, TDCA, GCA, GCDCA, and GDCA (Sigma-Aldrich) with molar rates of 12:12:8:23:23:16. The BSH activity of strains was expressed as the specific activity $(\mathrm{U} / \mathrm{mg}$ of protein).

For the plate and TLC assays, experiments were repeated twice, and results reported on the basis of direct visual observations as described above. For the colorimetric assay, experiments were repeated 3 times. Statistical analyses were performed using SPSS 15.0 software (SPSS Inc., Chicago, IL). Significant differences between treatments were tested by ANOVA followed by the Fisher least significant difference (LSD) test with a level of significance of $P<0.05$.

A TLC plate obtained with the use of this method for detection of BSH activity of strains is shown in Figure 1. For the uninoculated control sample (lane 2), a very weak CA spot appeared, which was the result of a slight impurity (CA) in the substrate sodium salts of GCA or TCA. The negative control strain (ATCC 25922, lane 8) and BSH-negative strains [F0122 (lane 3) and F0222 (lane 6)] produced a CA spot with an intensity similar to that of the uninoculated control sample. The positive control strain (ATCC 43121, lane 7) and BSH-positive strains [F0121 (lane 4) and F0221 (lane 5)] produced very robust CA spots on the plate as compared with the negative control or uninoculated control.

Forty-five lactobacilli were tested for BSH activity by the TLC, plate and quantitative colorimetric assays, and results are presented in Table 1. Results obtained from the TLC assay correlated well with that from the quantitative colorimetric assay. The same 24 strains were found to be BSH-positive with both methods. However, only 20 strains were found to be BSH-positive with the plate assay. Of the $24 \mathrm{BSH}$-positive strains as detected with the TLC assay, 4 strains (F0821, IN 3.21, IN 7.21, and F1623) failed to show BSH activity with the plate assay. Among these 4 strains, 3 (F0821, IN 3.21, IN 7.21) were found be BSH negative with the plate assay and for the remaining strain (F1623), 
BSH activity was undetectable because it was unable to grow on the MRS agar supplemented with sodium salt of TDCA.

To understand the basis for the discrepancy in results between the TLC and plate assays, the substrate specificity of the BSH from these 3 strains (F0821, IN 3.21 , IN 7.21) was assessed with the colorimetric assay using the 6 individual conjugated bile salts at a 9.4 $\mathrm{m} M$ substrate concentration as described by Tanaka et al. (2000). As shown in Table 2, all 3 strains lacked the ability to hydrolyze TDCA, whereas they were able to hydrolyze GCA and TCA, with the exception of strain F0821, which was able to hydrolyze GCA, but not TCA.

In the majority of cases, lactobacilli with BSH activity are able to hydrolyze simultaneously many types of human conjugated bile acids (Lundeen and Savage, 1990; Lambert et al., 2008). However, some exceptions to this generalization exist. Moser and Savage (2001) reported that L. buchneri JCM 1069 expressed BSH activity against TDCA, but not against TCA. Christiaens et al.(1992) found that L. brevis ATCC 14869 exhibited BSH activity toward GDCA, but not toward TDCA. These findings suggest that it is possible for certain BSH to recognize bile acids on both the cholate steroid nucleus and the amino acid groups. In the present study, strains IN3.21, IN7.21 and F0821were able to hydrolyze only bile acids with the CA, CA and CDCA, and glycin group, respectively (Table 2 ).

The BSH-positive strains detected by the TLC assay were the same as those detected by the quantitative colorimetric assay, indicating that all the BSH-positive strains were able to deconjugate GCA and TCA. Both GCA and TCA, as primary bile acids, predominate in the human bile as compared with TDCA, which exists as a secondary bile acid (Staggers et al., 1990). Thus, both GCA and TCA are often used as an indicator of human bile acids when studying bacterial BSH activity (Corzo and Gilliland, 1999a; Ramasamy et al., 2010). Although BSH activity is often found in intestinal lactobacilli, its exact biological significance is still unclear. Several hypotheses have been proposed suggesting that BSH activity benefits bacterium by enhancing its resistance to conjugated bile salts and increasing its survival in the gastrointestinal tract for colonization (Ridlon et al., 2006; Jones et al., 2008). The property that BSH-positive strains of lactobacilli prefer to deconjugate GCA or TCA may underlie their ability to enhance such potential benefits.

The finding that distinct spots can be yielded on TLC plates at very low levels of CA $(0.5 \mu \mathrm{g})$, indicates that BSH activity of cultures can be detected by the TLC assay with as little as $5 \%$ of substrate hydrolization (data not shown). Although spots of bile

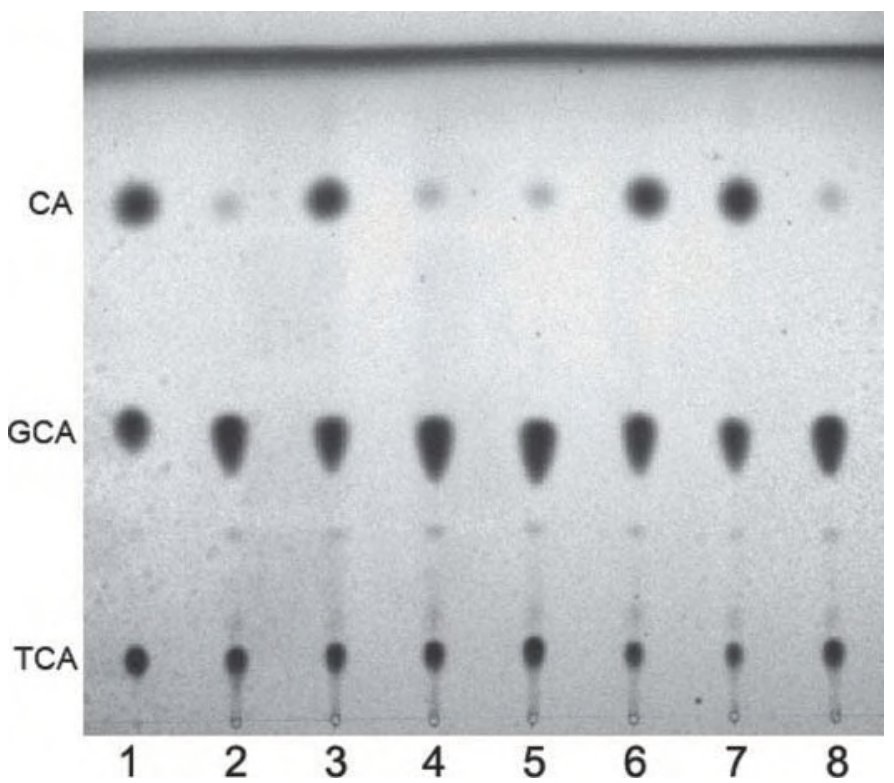

Figure 1. Typical pattern of bile salt hydrolase (BSH) activity from several strains of lactobacilli as detected by the thin-layer chromatography (TLC) assay. A solution of the samples was spotted manually on the TLC plate in a volume of $3 \mu \mathrm{L}$. Lane 1, mixed bile acid standard [glycocholic acid (GCA), taurocholic (TCA), and cholic acid (CA) in methanol, $5 \mathrm{mM}$ each); lane 2, uninoculated control (1 mL of sterile distilled water was added to the reaction mixture instead of the bacterial suspension); lane 3, strain F0122; lane 4, strain F0121; lane 5, strain F0221; lane 6, strain F0222; lane 7, strain ATCC 43121 (positive control strain); lane 8, strain ATCC 25922 (negative control strain).

acids can also be visualized by spraying the TLC plate with an aqueous solution of concentrated sulfuric acid (Eneroth, 1963), we found this approach to lack the sensitivity as that obtained with the ethanol solution of phosphomolybdic acid. The weakly corrosive chemical nature of phosphomolybdic acid makes it safer for use as a TLC visualization reagent, as opposed to sulfuric acid, which is a strongly corrosive chemical that can cause severe burns and tissue damage when contacting skin or mucous membranes. Given these considerations, phosphomolybdic acid was used as a visualization reagent in this study.

In humans, bile acid deconjugation mainly occurs in the ileum. The mean ileac $\mathrm{pH}$ is approximately $7.4(\mathrm{Ev}-$ ans et al., 1988; Ibekwe et al., 2008), and the mean bile acid concentration in the ileum is approximately $4 \mathrm{mM}$ with the ratio of glycoconjugates to tauroconjugates usually (2-3):1 (Liong and Shah, 2005b). The pH and concentration of the substrates used in the present TLC assay simulated physiological conditions of the human ileum. Accordingly, the results obtained from this in vitro test might similar to those from the in vivo test.

In conclusion, the TLC assay as described in this study was capable of detecting more BSH-positive 
Table 1. Comparison of different methods for detection of bile salt hydrolase (BSH) activity of Lactobacillus strains

\begin{tabular}{|c|c|c|c|}
\hline \multirow[b]{3}{*}{ Strain code } & \multicolumn{3}{|c|}{ BSH activity } \\
\hline & \multicolumn{2}{|c|}{ Qualitative assay } & \multirow{2}{*}{$\begin{array}{l}\text { Quantitative assay } \\
\begin{array}{l}\text { Colorimetric assay } \\
\text { (U/mg of protein) }\end{array}\end{array}$} \\
\hline & $\begin{array}{l}\text { Plate } \\
\text { assay }^{1}\end{array}$ & $\begin{array}{l}\text { TLC } \\
\text { assay }^{2}\end{array}$ & \\
\hline F1022 & $\mathrm{P}(\mathrm{p})$ & $\mathrm{P}$ & $2.00 \pm 0.34^{*}$ \\
\hline F1421 & $\mathrm{P}(\mathrm{p})$ & $\mathrm{P}$ & $1.82 \pm 0.19^{*}$ \\
\hline F0521 & $\mathrm{P}(\mathrm{p})$ & $\mathrm{P}$ & $1.81 \pm 0.33^{*}$ \\
\hline F0822 & $\mathrm{P}(\mathrm{p})$ & $\mathrm{P}$ & $1.63 \pm 0.36^{*}$ \\
\hline F1821 & $\mathrm{P}(\mathrm{w})$ & $\mathrm{P}$ & $1.47 \pm 0.27^{*}$ \\
\hline IN2.22 & $\mathrm{P}(\mathrm{p})$ & $\mathrm{P}$ & $1.40 \pm 0.21^{*}$ \\
\hline F0821 & $\mathrm{N}$ & $\mathrm{P}$ & $1.30 \pm 0.24^{*}$ \\
\hline IN5.22 & $\mathrm{P}(\mathrm{p})$ & $\mathrm{P}$ & $1.25 \pm 0.14^{*}$ \\
\hline F0422 & $\mathrm{P}(\mathrm{p})$ & $\mathrm{P}$ & $1.08 \pm 0.17^{*}$ \\
\hline F0122 & $\mathrm{P}(\mathrm{p})$ & $\mathrm{P}$ & $0.93 \pm 0.17^{*}$ \\
\hline F0121 & $\mathrm{P}(\mathrm{p})$ & $\mathrm{P}$ & $0.85 \pm 0.20^{*}$ \\
\hline F1722 & $\mathrm{P}(\mathrm{p})$ & $\mathrm{P}$ & $0.80 \pm 0.17^{*}$ \\
\hline IN1.21 & $\mathrm{P}(\mathrm{w})$ & $\mathrm{P}$ & $0.74 \pm 0.19^{*}$ \\
\hline IN3.21 & $\mathrm{N}$ & $\mathrm{P}$ & $0.68 \pm 0.07^{*}$ \\
\hline F1222 & $\mathrm{P}(\mathrm{p})$ & $\mathrm{P}$ & $0.59 \pm 0.14^{*}$ \\
\hline IN2.21 & $\mathrm{P}(\mathrm{p})$ & $\mathrm{P}$ & $0.56 \pm 0.14^{*}$ \\
\hline F0921 & $\mathrm{P}(\mathrm{p})$ & $\mathrm{P}$ & $0.52 \pm 0.09^{*}$ \\
\hline F0621 & $\mathrm{P}(\mathrm{w})$ & $\mathrm{P}$ & $0.46 \pm 0.07^{*}$ \\
\hline IN6.21 & $\mathrm{P}(\mathrm{p})$ & $\mathrm{P}$ & $0.39 \pm 0.07^{*}$ \\
\hline F1623 & 0 & $\mathrm{P}$ & $0.39 \pm 0.07^{*}$ \\
\hline IN7.21 & $\mathrm{N}$ & $\mathrm{P}$ & $0.35 \pm 0.07^{*}$ \\
\hline F1121 & $\mathrm{P}(\mathrm{w})$ & $\mathrm{P}$ & $0.33 \pm 0.06^{*}$ \\
\hline IN9.22 & $\mathrm{P}(\mathrm{w})$ & $\mathrm{P}$ & $0.24 \pm 0.05^{*}$ \\
\hline F0222 & $\mathrm{P}(\mathrm{w})$ & $\mathrm{P}$ & $0.23 \pm 0.04^{*}$ \\
\hline IN3.22 & $\mathrm{N}$ & $\mathrm{N}$ & $0.03 \pm 0.01$ \\
\hline F1721 & $\mathrm{N}$ & $\mathrm{N}$ & $0.03 \pm 0.02$ \\
\hline IN6.22 & $\mathrm{N}$ & $\mathrm{N}$ & $0.03 \pm 0.01$ \\
\hline F0922 & $\mathrm{N}$ & $\mathrm{N}$ & $0.02 \pm 0.01$ \\
\hline F1322 & $\mathrm{N}$ & $\mathrm{N}$ & $0.02 \pm 0.02$ \\
\hline F0421 & $\mathrm{N}$ & $\mathrm{N}$ & $0.02 \pm 0.02$ \\
\hline IN5.21 & $\mathrm{N}$ & $\mathrm{N}$ & $0.02 \pm 0.03$ \\
\hline F1522 & 0 & $\mathrm{~N}$ & $0.02 \pm 0.02$ \\
\hline IN4.21 & $\mathrm{N}$ & $\mathrm{N}$ & $0.02 \pm 0.03$ \\
\hline F0622 & $\mathrm{N}$ & $\mathrm{N}$ & $0.02 \pm 0.01$ \\
\hline F1321 & $\mathrm{N}$ & $\mathrm{N}$ & $0.02 \pm 0.03$ \\
\hline F1523 & $\mathrm{N}$ & $\mathrm{N}$ & $0.01 \pm 0.02$ \\
\hline IN9.21 & 0 & $\mathrm{~N}$ & $0.01 \pm 0.02$ \\
\hline F1622 & $\mathrm{N}$ & $\mathrm{N}$ & $0.01 \pm 0.03$ \\
\hline F0221 & $\mathrm{N}$ & $\mathrm{N}$ & $0.01 \pm 0.03$ \\
\hline IN1.22 & 0 & $\mathrm{~N}$ & $0.01 \pm 0.03$ \\
\hline F1521 & $\mathrm{N}$ & $\mathrm{N}$ & $0.01 \pm 0.03$ \\
\hline F1021 & $\mathrm{N}$ & $\mathrm{N}$ & $0.00 \pm 0.03$ \\
\hline F0321 & $\mathrm{N}$ & $\mathrm{N}$ & $-0.01 \pm 0.01$ \\
\hline F1221 & $\mathrm{N}$ & $\mathrm{N}$ & $-0.01 \pm 0.03$ \\
\hline F0721 & $\mathrm{N}$ & $\mathrm{N}$ & $-0.01 \pm 0.02$ \\
\hline ATCC 43121 & $\mathrm{P}(\mathrm{w})$ & $\mathrm{P}$ & $1.76 \pm 0.25^{*}$ \\
\hline ATCC 25922 & 0 & $\mathrm{~N}$ & $0.01 \pm 0.02$ \\
\hline
\end{tabular}

${ }^{1}$ Capacity to express taurodeoxycholic acid (TDCA) hydrolase activity as detected when precipitates of deoxycholic acid appeared below bacterial colonies growing on de Man, Rogosa, and Sharpe (MRS)-TDCA. $0=$ no growth; $\mathrm{N}=$ negative; $\mathrm{P}(\mathrm{p})=$ positive $\mathrm{BSH}$ activity and formation of precipitate halos around colonies; $\mathrm{P}(\mathrm{w})$ $=$ positive BSH activity and formation and opaque granular white colonies.

${ }^{2}$ Thin-layer chromatography capacity to express glycocholic acid (GCA) and taurocholic acid (TCA) hydrolase activity as detected when visible spots of cholic acid (CA) appeared on the thin-layer plates compared with the control. $\mathrm{P}=$ positive; $\mathrm{N}=$ negative.

${ }^{3}$ Results are presented as mean \pm SD of BSH activity expressed as specific activity.

*Asterisks in the same column indicate statistically significant differences $(P<0.05)$ between the tested and negative control (ATCC 25922) strains. 
Table 2. Substrate specificity of 3 isolates toward the 6 individual human conjugated bile salts

\begin{tabular}{|c|c|c|c|c|}
\hline \multirow[b]{2}{*}{$\begin{array}{l}\text { Conjugated } \\
\text { bile salt }\end{array}$} & \multicolumn{4}{|c|}{$\mathrm{BSH}_{\operatorname{activity}^{2}}$ (U/mg of protein) } \\
\hline & $\begin{array}{l}\text { Strain } \\
\text { F0821 }\end{array}$ & $\begin{array}{l}\text { Strain } \\
\text { IN3.21 }\end{array}$ & $\begin{array}{l}\text { Strain } \\
\text { IN7.21 }\end{array}$ & $\begin{array}{c}\text { Strain } \\
\text { ATCC } 25922\end{array}$ \\
\hline GCA & $0.90 \pm 0.12^{*}$ & $0.53 \pm 0.11^{*}$ & $0.25 \pm 0.07^{*}$ & $0.01 \pm 0.01$ \\
\hline GCDCA & $0.73 \pm 0.17^{*}$ & $0.01 \pm 0.03$ & $0.85 \pm 0.19^{*}$ & $0.00 \pm 0.02$ \\
\hline GDCA & $0.41 \pm 0.12^{*}$ & $-0.01 \pm 0.02$ & $0.01 \pm 0.01$ & $-0.01 \pm 0.02$ \\
\hline TCA & $0.02 \pm 0.01$ & $0.26 \pm 0.09^{*}$ & $0.22 \pm 0.08^{*}$ & $0.02 \pm 0.01$ \\
\hline TCDCA & $0.01 \pm 0.02$ & $0.02 \pm 0.01$ & $0.51 \pm 0.10^{*}$ & $0.00 \pm 0.02$ \\
\hline TDCA & $0.02 \pm 0.01$ & $0.01 \pm 0.02$ & $0.02 \pm 0.01$ & $0.01 \pm 0.02$ \\
\hline
\end{tabular}

${ }^{1} \mathrm{GCA}=$ glycocholic acid; GCDCA = glycochenodeoxycholic acid; GDCA = glycodeoxycholic acid; TCA = taurocholic acid; TCDCA = taurochenodeoxycholic acid; TDCA = taurodeoxycholic acid.

${ }^{2}$ Results are presented as mean \pm SD of bile salt hydrolase (BSH) activity expressed as specific activity. The asterisk $(*)$ in the same row indicates statistically significant differences $(P<0.05)$ between the tested and negative control (ATCC 25922) strains.

strains than that with use of the conventional plate assay. As a result, this TLC assay possesses a higher sensitivity for screening of BSH-active lactobacilli. In addition, unlike the plate assay, this TLC assay does not require that strains grow in the presence of bile salts, and, thus, has a wider application range than the plate assay.

\section{ACKNOWLEDGMENTS}

This work was financially supported by National High-Tech R\&D Program Grants from the Ministry of Science and Technology of the People's Republic of China (2007AA10Z354, 2006BAD04A06, 2006BAD04A09) and R\&D Program Grants from the Educational Committee of Heilongjiang Province (1055GZD03-2004).

\section{REFERENCES}

Begley, M., C. Hill, and C. G. M. Gahan. 2006. Bile salt hydrolase activity in probiotics. Appl. Environ. Microbiol. 72:1729-1738.

Brashears, M. M., S. E. Gilliland, and L. M. Buck. 1998. Bile salt deconjugation and cholesterol removal from media by Lactobacillus casei. J. Dairy Sci. 81:2103-2110.

Christiaens, H., R. J. Leer, P. H. Pouwels, and W. Verstraete. 1992. Cloning and expression of a conjugated bile acid hydrolase gene from Lactobacillus plantarum by using a direct plate assay. Appl. Environ. Microbiol. 58:3792-3798.

Cole, C. B., and R. Fuller. 1984. Bile acid deconjugation and attachment of chicken gut bacteria: Their possible role in growth depression. Br. Poult. Sci. 25:227-231.

Corzo, G., and S. E. Gilliland. 1999a. Bile salt hydrolase activity of three strains of Lactobacillus acidophilus. J. Dairy Sci. 82:472480.

Corzo, G., and S. E. Gilliland. 1999b. Measurement of bile salt hydrolase activity from Lactobacillus acidophilus based on disappearance of conjugated bile salts. J. Dairy Sci. 82:466-471.

Dashkevicz, M. P., and S. D. Feighner. 1989. Development of a differential medium for bile-salt hydrolase-active Lactobacillus spp. Appl. Environ. Microbiol. 55:11-16.

De Smet, I., P. De Boever, and W. Verstraete. 1998. Cholesterol lowering in pigs through enhanced bacterial bile salt hydrolase activity. Br. J. Nutr. 79:185-194.
Eneroth, P. 1963. Thin-layer chromatography of bile acids. J. Lipid Res. 4:11-16.

Evans, D. F., G. Pye, R. Bramley, A. G. Clark, T. J. Dyson, and J. D. Hardcastle. 1988. Measurement of gastrointestinal $\mathrm{pH}$ profiles in normal ambulant human subjects. Gut 29:1035-1041.

Fang, F., Y. Li, M. Bumann, E. J. Raftis, P. G. Casey, J. C. Cooney, M. A. Walsh, and P. W. O'Toole. 2009. Allelic variation of bile salt hydrolase genes in Lactobacillus salivarius does not determine bile resistance levels. J. Bacteriol. 191:5743-5757.

Hartemink, R., V. R. Domenech, and F. M. Rombouts. 1997. LAMV$\mathrm{AB}-\mathrm{A}$ new selective medium for the isolation of lactobacilli from faeces. J. Microbiol. Methods 29:77-84.

Hofmann, A. F. 1962. Thin-layer adsorption chromatography of free and conjugated bile acids on silicic acid. J. Lipid Res. 3:127128.

Ibekwe, V. C., H. M. Fadda, E. L. McConnell, M. K. Khela, D. F. Evans, and A. W. Basit. 2008. Interplay between intestinal pH, transit time and feed status on the in vivo performance of $\mathrm{pH}$ responsive ileo-colonic release systems. Pharm. Res. 25:1828-1835.

Jones, B. V., M. Begley, C. Hill, C. G. M. Gahan, and J. R. Marchesi. 2008. Functional and comparative metagenomic analysis of bile salt hydrolase activity in the human gut microbiome. Proc. Natl. Acad. Sci. USA 105:13580-13585.

Kim. G.-B., S.-H. Yi, and B. H. Lee. 2004. Purification and characterization of three different types of bile salt hydrolases from Bifidobacterium strains. J. Dairy Sci. 87:258-266.

Lambert, J. M., R. S. Bongers, W. M. de Vos, and M. Kleerebezem. 2008. Functional analysis of four bile salt hydrolase and penicillin acylase family members in Lactobacillus plantarum WCFS1. Appl. Environ. Microbiol. 74:4719-4726.

Lebeer, S., J. Vanderleyden, and S. C. J. De Keersmaecker. 2008. Genes and molecules of lactobacilli supporting probiotic action. Microbiol. Mol. Biol. Rev. 72:728-764.

Liong, M. T., and N. P. Shah. 2005a. Bile salt deconjugation ability, bile salt hydrolase activity and cholesterol co-precipitation ability of lactobacilli strains. Int. Dairy J. 15:391-398.

Liong, M. T., and N. P. Shah. 2005b. Bile salt deconjugation and BSH activity of five bifidobacterial strains and their cholesterol co-precipitating properties. Food Res. Int. 38:135-142.

Lundeen, S. G., and D. C. Savage. 1990. Characterization and purification of bile salt hydrolase from Lactobacillus sp. strain 100-100. J. Bacteriol. 172:4171-4177.

Moser, S. A., and D. C. Savage. 2001. Bile salt hydrolase activity and resistance to toxicity of conjugated bile salts are unrelated properties in lactobacilli. Appl. Environ. Microbiol. 67:3476-3480.

Nguyen, T. D. T., J. H. Kang, and M. S. Lee. 2007. Characterization of Lactobacillus plantarum $\mathrm{PH} 04$, a potential probiotic bacterium with cholesterol-lowering effects. Int. J. Food Microbiol. 113:358-361. 
Noriega, L., I. Cuevas, A. Margolles, and C. G. de los Reyes-Gavilán. 2006. Deconjugation and bile salts hydrolase activity by Bifidobacterium strains with acquired resistance to bile. Int. Dairy J. 16:850-855.

Pereira, D. I. A., A. L. McCartney, and G. R. Gibson. 2003. An in vitro study of the probiotic potential of a bile-salt-hydrolyzing Lactobacillus fermentum strain, and determination of its cholesterollowering properties. Appl. Environ. Microbiol. 69:4743-4752.

Ramasamy, K., N. Abdullah, M. C. V. L. Wong, C. Karuthan, and Y. W. Ho. 2010. Bile salt deconjugation and cholesterol removal from media by Lactobacillus strains used as probiotics in chickens. J. Sci. Food Agric. 90:65-69.

Ridlon, J. M., D. J. Kang, and P. B. Hylemon. 2006. Bile salt biotransformations by human intestinal bacteria. J. Lipid Res. $47: 241-259$.
Staggers, J. E., O. Hernell, R. J. Stafford, and M. C. Carey. 1990. Physical-chemical behavior of dietary and biliary lipids during intestinal digestion and absorption. 1. Phase behavior and aggregation states of model lipid systems patterned after aqueous duodenal contents of healthy adult human beings. Biochemistry 29:2028-2040.

Tanaka, H., H. Hashiba, J. Kok, and I. Mierau. 2000. Bile salt hydrolase of Bifidobacterium longum - Biochemical and genetic characterization. Appl. Environ. Microbiol. 66:2502-2512.

Walker, D. K., and S. E. Gilliland. 1993. Relationships among bile tolerance, bile salt deconjugation, and assimilation of cholesterol by Lactobacillus acidophilus. J. Dairy Sci. 76:956-961. 